\title{
Archives
}

\section{Index du volume 46 (2016-2017)}

VIVIANNE MARÉCHAL

Volume 46, numéro 2, 2017

URI : https://id.erudit.org/iderudit/1040390ar

DOI : https://doi.org/10.7202/1040390ar

Aller au sommaire du numéro

Éditeur(s)

Association des archivistes du Québec (AAQ)

ISSN

0044-9423 (imprimé)

2369-9256 (numérique)

Découvrir la revue

Citer ce document

Maréchal, V. (2017). Index du volume 46 (2016-2017). Archives, 46(2), 191-200.

https://doi.org/10.7202/1040390ar

Ce document est protégé par la loi sur le droit d'auteur. L’utilisation des services d'Érudit (y compris la reproduction) est assujettie à sa politique d'utilisation que vous pouvez consulter en ligne.

https://apropos.erudit.org/fr/usagers/politique-dutilisation/
Cet article est diffusé et préservé par Érudit.

Érudit est un consortium interuniversitaire sans but lucratif composé de l’Université de Montréal, l'Université Laval et l'Université du Québec à Montréal. Il a pour mission la promotion et la valorisation de la recherche. https://www.erudit.org/fr/ 


\section{ANNIVERSAIRE DE LA DGDA}

\section{Index du volume 46 (2016-2017)}

\section{VIVIANNE MARÉCHAL}

L'index du volume 46 de la revue Archives se divise en trois parties: un index de chaque auteur de tout article ou compte rendu, un index alphabétique des sujets ainsi qu'un index des ouvrages ayant fait l'objet d'un compte rendu critique. Un ouvrage écrit par plusieurs auteurs se retrouve indexé pour chaque auteur. De plus, chaque article est indexé de manière à ce que sa notice bibliographique apparaisse au moins sous deux mots-clés différents dans l'index des sujets. Finalement, les ouvrages recensés se retrouvent dans l'index des auteurs, sous le nom de l'auteur du compte rendu, ainsi que dans l'index des ouvrages recensés sous le nom de l'auteur de l'ouvrage.

\section{INDEX DES AUTEURS}

BAILLARGEON, D. (2017). L'apport de François Beaudin: la mise en place du rapport Deschênes. 46(2), 15-26.

BAILLARGEON, D. Les prochains défis de la Division de la gestion de documents et des archives de l'Université de Montréal. 46(2), 163-175.

BELIN, A. (2016). Archivage électronique et analyse de risque: Les nouveaux défis de l'archiviste. 46(1), 47-60. 
BOUDREAU, D. (2016). Diffuser, partager et s'approprier le patrimoine documentaire québécois. Le projet collaboratif de BAnQ sur Wikimédia: une première au Canada. 46(1), 61-82.

BOURHIS, Taïk. (2017). La gestion des archives courantes et intermédiaires: la Division de la gestion de documents et des archives de I'Université de Montréal au rythme de sa clientèle. 46(2), 35-60.

BROCHU, F. (2017). L'harmonisation des principes et des méthodes des sciences de l'information: I'approche gagnante d'une pratique professionnelle de l'archivistique (Témoignage). 46(2), 113-118.

BUTHIAUX, A. (2016). [Compte rendu de I'ouvrage Archives and Recordkeeping: Theory Into Practice, par P. Whatley et C. Brown]. 46(1), 101-106.

CHAMPAGNE, M. (2017). Les archives définitives à la Division de la gestion de documents et des archives de l'Université de Montréal: accroissement, traitement, diffusion. 46(2), 61-80.

CHOUINARD, D. (2017). L'influence du Service des archives de l'Université de Montréal sur la pratique archivistique au Québec: une affaire de fierté (Témoignage). 46(2), 119-122.

DA SILVA PRADO, D. (2016). L'archivistique au Brésil: points de repère. 46(1), 5-14.

DAVEAU, F. (2016). Diffuser, partager et s'approprier le patrimoine documentaire québécois. Le projet collaboratif de BAnQ sur Wikimédia: une première au Canada. 46(1), 61-82.

DENIS, N. (2016). La gestion des archives courantes et intermédiaires: la Division de la gestion de documents et des archives de l'Université de Montréal au rythme de sa clientèle. 46(2), 35-60.

DION, I. (2017). De la théorie à la pratique: I'exemple des stages de la Division de la gestion de documents et des archives de l'Université de Montréal. 46(2), 91-112.

DUFRESNE, M.-C. (2016). Réduire les silos d'information - Bilan d'expérience au Cirque du Soleil avec la gestion électronique des documents. 46(1), 83-100. 
DUGAS, C. (2017). La Division de la gestion de documents et des archives de I'Université de Montréal et l'Association des archivistes du Québec: des chemins croisés. 46(2), 133-143.

GIULIANO, F. (2016). Diffuser, partager et s'approprier le patrimoine documentaire québécois. Le projet collaboratif de BAnQ sur Wikimédia: une première au Canada. 46(1), 61-82.

GRATTON, E. (2016). Réduire les silos d'information - Bilan d'expérience au Cirque du Soleil avec la gestion électronique des documents. 46(1), 83-100.

LAJEUNESSE, M. (2017). Le Service des archives de I'Université de Montréal et la formation des archivistes. 46(2), 81-90.

LALONDE, M. (2017). Le Service des archives de I'Université de Montréal: une formidable école! (Témoignage). 46(2), 123-132.

LAVERDURE, H. (2017). L'influence de la Division de la gestion de documents et des archives de l'Université de Montréal sur la pratique archivistique nationale. 46(2), 145-150.

LESPÉRANCE, M. (2017). Bref historique des grandes réalisations de la Division de la gestion de documents et des archives de l'Université de Montréal (1966-2016). 46(2), 7-14.

MAS, S. (2016). [Compte rendu de l'ouvrage Regards croisés sur les métiers des sciences de l'information: Bibliothèques, Archives, Documentation, Musée, par J.-P. Accart]. 46(1), 107-109.

MINOTTO, C. (2017). La mémoire utile, fond et forme au menu des années 2000. 46(2), 27-33.

RIETSCH, J.-M. (2016). Archivage électronique et analyse de risque: Les nouveaux défis de l'archiviste. 46(1), 47-60.

SANSOM, C. (2016). [Compte rendu de I'ouvrage Identity Palimpsests: Archiving Ethnicity in the U.S. and Canada, par D. Daniel et A. Levi (dir.)]. 46(1), 111-113.

SCARPULLA, M. (2016). Archives, danse et recréation. Une introduction. $46(1), 15-34$. 
SERVAIS, P. (2017). La Division de la gestion de documents et des archives de I'Université de Montréal: un regard d'Outre-Atlantique. 46(2), 151-161.

WINAND, A. (2016). Archives et réemploi dans les films expérimentaux. 46(1), 35-46.

ZELLER, J.-D. (2016). [Compte rendu de l'ouvrage L'archivistique à l'ère du numérique - Les éléments fondamentaux de la discipline par C. Couture et M. Lajeunesse]. 46(1), 115-124.

\section{INDEX DES SUJETS}

\section{Acquisition}

CHAMPAGNE, M. (2017). Les archives définitives à la Division de la gestion de documents et des archives de I'Université de Montréal: accroissement, traitement, diffusion. 46(2), 61-80.

\section{Administration}

BAILLARGEON, D. (2017). Les prochains défis de la Division de la gestion de documents et des archives de I'Université de Montréal. 46(2), 163-175.

MINOTTO, C. (2017). La mémoire utile, fond et forme au menu des années 2000. 46(2), 27-33.

\section{Analyse documentaire}

CHAMPAGNE, M. (2017). Les archives définitives à la Division de la gestion de documents et des archives de l'Université de Montréal: accroissement, traitement, diffusion. 46(2), 61-80.

BOUDREAU, D., DAVEAU, F. et GIULIANO, F. (2016). Diffuser, partager et $s$ 'approprier le patrimoine documentaire québécois. Le projet collaboratif de BAnQ sur Wikimédia: une première au Canada. 46(1), 61-82.

\section{Archives courantes}

BOURHIS, T. et DENIS, N. (2017). La gestion des archives courantes et intermédiaires: la Division de la gestion de documents et des archives de I'Université de Montréal au rythme de sa clientèle. 46(2), 35-60. 
DUFRESNE, M.-C. et GRATTON, E. (2016). Réduire les silos d'information - Bilan d'expérience au Cirque du Soleil avec la gestion électronique des documents. 46(1), 83-100.

SCARPULLA, M. (2016). Archives, danse et recréation. Une introduction. 46(1), 15-34.

\section{Archives définitives}

CHAMPAGNE, M. (2017). Les archives définitives à la Division de la gestion de documents et des archives de I'Université de Montréal: accroissement, traitement, diffusion. 46(2), 61-80.

SCARPULLA, M. (2016). Archives, danse et recréation. Une introduction. $46(1), 15-34$.

\section{Archives intermédiaires}

BOURHIS, T. et DENIS, N. (2017). La gestion des archives courantes et intermédiaires: la Division de la gestion de documents et des archives de I'Université de Montréal au rythme de sa clientèle. 46(2), 35-60.

\section{Brésil}

DA SILVA PRADO, D. (2016). L'archivistique au Brésil: points de repère. 46(1), 5-14.

\section{Clients}

BOURHIS, T. et DENIS, N. (2017). La gestion des archives courantes et intermédiaires: la Division de la gestion de documents et des archives de I'Université de Montréal au rythme de sa clientèle. 46(2), 35-60.

\section{Création}

WINAND, A. (2016). Archives et réemploi dans les films expérimentaux. 46(1), 35-46.

\section{Culture}

BOUDREAU, D., DAVEAU, F. et GIULIANO, F. (2016). Diffuser, partager et s'approprier le patrimoine documentaire québécois. Le projet collaboratif de BAnQ sur Wikimédia: une première au Canada. 46(1), 61-82. 
LAVERDURE, H. (2017). L'influence de la Division de la gestion de documents et des archives de l'Université de Montréal sur la pratique archivistique nationale. 46(2), 145-150.

SCARPULLA, M. (2016). Archives, danse et recréation. Une introduction. 46(1), 15-34.

WINAND, A. (2016). Archives et réemploi dans les films expérimentaux. $46(1), 35-46$.

\section{Diffusion}

BOUDREAU, D., DAVEAU, F. et GIULIANO, F. (2016). Diffuser, partager et s'approprier le patrimoine documentaire québécois. Le projet collaboratif de BAnQ sur Wikimédia: une première au Canada. 46(1), 61-82.

CHAMPAGNE, M. (2017). Les archives définitives à la Division de la gestion de documents et des archives de l'Université de Montréal: accroissement, traitement, diffusion. 46(2), 61-80.

SCARPULLA, M. (2016). Archives, danse et recréation. Une introduction. 46(1), 15-34.

\section{Documents audiovisuels}

WINAND, A. (2016). Archives et réemploi dans les films expérimentaux. 46(1), 35-46.

\section{Documents informatiques}

BELIN, A. et RIETSCH, J.-M. (2016). Archivage électronique et analyse de risque: Les nouveaux défis de l'archiviste. 46(1), 47-60.

BOURHIS, T. et DENIS, N. (2017). La gestion des archives courantes et intermédiaires: la Division de la gestion de documents et des archives de I'Université de Montréal au rythme de sa clientèle. 46(2), 35-60.

MINOTTO, C. (2017). La mémoire utile, fond et forme au menu des années 2000. 46(2), 27-33.

\section{Éducation}

BAILLARGEON, D. (2017). L'apport de François Beaudin : la mise en place du rapport Deschênes. 46(2), 15-26. 
BAILLARGEON, D. (2017). Les prochains défis de la Division de la gestion de documents et des archives de I'Université de Montréal. 46(2), 163-175.

BOURHIS, T. et DENIS, N. (2017). La gestion des archives courantes et intermédiaires: la Division de la gestion de documents et des archives de l'Université de Montréal au rythme de sa clientèle. 46(2), 35-60.

BROCHU, F. (2017). L'harmonisation des principes et des méthodes des sciences de l'information: I'approche gagnante d'une pratique professionnelle de l'archivistique (Témoignage). 46(2), 113-118.

CHAMPAGNE, M. (2017). Les archives définitives à la Division de la gestion de documents et des archives de l'Université de Montréal: accroissement, traitement, diffusion. 46(2), 61-80.

CHOUINARD, D. (2017). L'influence du Service des archives de l'Université de Montréal sur la pratique archivistique au Québec: une affaire de fierté (Témoignage). 46(2), 119-122.

DION, I. (2017). De la théorie à la pratique: I'exemple des stages de la Division de la gestion de documents et des archives de l'Université de Montréal. 46(2), 91-112.

DUGAS, C. (2017). La Division de la gestion de documents et des archives de l'Université de Montréal et l'Association des archivistes du Québec: des chemins croisés. 46(2), 133-143.

LAJEUNESSE, M. (2017). Le Service des archives de I'Université de Montréal et la formation des archivistes. 46(2), 81-90.

LALONDE, M. (2017). Le Service des archives de I'Université de Montréal: une formidable école! (Témoignage). 46(2), 123-132.

LAVERDURE, H. (2017). L'influence de la Division de la gestion de documents et des archives de l'Université de Montréal sur la pratique archivistique nationale. 46(2), 145-150.

LESPÉRANCE, M. (2017). Bref historique des grandes réalisations de la Division de la gestion de documents et des archives de I'Université de Montréal (1966-2016). 46(2), 7-14. 
MINOTTO, C. (2017). La mémoire utile, fond et forme au menu des années 2000. 46(2), 27-33.

SERVAIS, P. (2017). La Division de la gestion de documents et des archives de I'Université de Montréal: un regard d'Outre-Atlantique. 46(2), 151-161.

\section{Formation}

DION, I. (2017). De la théorie à la pratique: I'exemple des stages de la Division de la gestion de documents et des archives de I'Université de Montréal. 46(2), 91-112.

LAJEUNESSE, M. (2017). Le Service des archives de I'Université de Montréal et la formation des archivistes. 46(2), 81-90.

LALONDE, M. (2017). Le Service des archives de I'Université de Montréal: une formidable école! (Témoignage). 46(2), 123-132.

\section{Histoire}

BAILLARGEON, D. (2017). L'apport de François Beaudin : la mise en place du rapport Deschênes. 46(2), 15-26.

LESPÉRANCE, M. (2017). Bref historique des grandes réalisations de la Division de la gestion de documents et des archives de l'Université de Montréal (1966-2016). 46(2), 7-14.

\section{Informatisation}

BELIN, A. et RIETSCH, J.-M. (2016). Archivage électronique et analyse de risque: Les nouveaux défis de l'archiviste. 46(1), 47-60.

DUFRESNE, M.-C. et GRATTON, E. (2016). Réduire les silos d'information - Bilan d'expérience au Cirque du Soleil avec la gestion électronique des documents. 46(1), 83-100.

\section{Législation}

DA SILVA PRADO, D. (2016). L'archivistique au Brésil: points de repère. 46(1), 5-14. 


\section{Organisation archivistique}

DUGAS, C. (2017). La Division de la gestion de documents et des archives de I'Université de Montréal et l'Association des archivistes du Québec: des chemins croisés. 46(2), 133-143.

\section{Profession}

BROCHU, F. (2017). L'harmonisation des principes et des méthodes des sciences de l'information: I'approche gagnante d'une pratique professionnelle de l'archivistique (Témoignage). 46(2), 113-118.

CHOUINARD, D. (2017). L'influence du Service des archives de l'Université de Montréal sur la pratique archivistique au Québec: une affaire de fierté (Témoignage). 46(2), 119-122.

DION, I. (2017). De la théorie à la pratique: I'exemple des stages de la Division de la gestion de documents et des archives de l'Université de Montréal. 46(2), 91-112.

DUGAS, C. (2017). La Division de la gestion de documents et des archives de I'Université de Montréal et l'Association des archivistes du Québec: des chemins croisés. 46(2), 133-143.

LAJEUNESSE, M. (2017). Le Service des archives de I'Université de Montréal et la formation des archivistes. 46(2), 81-90.

LALONDE, M. (2017). Le Service des archives de I'Université de Montréal: une formidable école! (Témoignage). 46(2), 123-132.

LAVERDURE, H. (2017). L'influence de la Division de la gestion de documents et des archives de l'Université de Montréal sur la pratique archivistique nationale. 46(2), 145-150.

MINOTTO, C. (2017). La mémoire utile, fond et forme au menu des années 2000. 46(2), 27-33.

SERVAIS, P. (2017). La Division de la gestion de documents et des archives de l'Université de Montréal: un regard d'Outre-Atlantique. 46(2), 151-161. 


\section{Secteur privé}

DUFRESNE, M.-C. et GRATTON, E. (2016). Réduire les silos d'information - Bilan d'expérience au Cirque du Soleil avec la gestion électronique des documents. 46(1), 83-100.

\section{INDEX DES OUVRAGES RECENCÉS}

ACCART, J.-P. (2014). Regards croisés sur les métiers des sciences de I'information: Bibliothèques, Archives, Documentation, Musées. MontSaint-Aignan: éditions KLOG, 119 p. (Compte rendu par S. Mas, 46(1), 107-109)

COUTURE, C. et LAJEUNESSE, M. (2015). L'archivistique à l'ère du numérique - Les éléments fondamentaux de la discipline. Québec: Les Presses de l'Université du Québec, 278 p. (Compte rendu par J.-D. Zeller, 46(1), 115-124)

DANIEL, D. et A. LEVI, dir. (2014). Identity Palimpsests: Archiving Ethnicity in the U.S. and Canada. Sacramento, C.A. L Litwin Books. (Compte rendu par C. Samson, 46(1), 111-113)

WHATLEY, P. et C. BROWN. (2013). Archives and Recordkeeping: Theory Into Practice. London: Facet Publishing, 260 p. (Compte rendu par A. Buthiaux, 46(1), 101-106) 